\title{
XXII. On secondary radiation
}

\section{J.A. McClelland M.A.}

To cite this article: J.A. McClelland M.A. (1905) XXII. On secondary radiation , Philosophical Magazine Series 6, 9:50, 230-243, DOI: 10.1080/14786440509463274

To link to this article: http://dx.doi.org/10.1080/14786440509463274

册 Published online: 08 Jun 2010.

Submit your article to this journal

Џ Article views: 5

Q View related articles $\sqsubset$ 


\section{[ 230$]$}

XXII. On Secondary Radiation. By J. A. McCleblann, M.A., Professor of Experimental Pliysics, University College, Dublin*.

THE following paper gives an account of an investigation of the secondary radiation given off by bodies when they are exposed to a strong primary radiation from radium.

In the present paper, the secondary radiation produced by the more penetrating radium rays only-the $\beta$ and $\gamma$ rays- has been considered; the nature of this secondary radiation and its relative intensity in the case of different substances have been studied. The subject of secondary radiation seems of some importance and promise, as there are many problems on which it may have a possible bearing ; it is elosely related to the subject of spontaneous radioactivity and to all phenomena produced by the discharge from bodies of particles charged with electricity.

Secondary radiation under the action of catbode rays bas been investigated by Starke $\dagger$, Austin and Starke $\neq$, Swinton $\S$, and others. Secondary radiation under the action of Röntgen rays has been studied by Perrin \|. Townsend $\mathbb{T}$, and others; while secondary radiation due to radium rays has been cietected and investigated to some extent by Becquerel ** and others. $\dagger^{\dagger}$

\section{Apparatus.}

The secoudary radiation was detected and measured by its ionizing power. Fig. 1 gives a sketch of the apparatus used. $\mathrm{T}$ is a brass tube $20 \mathrm{cms}$. in length and $3 \cdot 2 \mathrm{cms}$. internal diameter, connected to small storage-cells by which it could be kept at any required potential ; a metal rod is fixed along the axis of the tube, being insulated by paraffin and joined to a Dolezalek electrometer, the joining wire being suitably

* Communicated by the Author. From Royal Dublin Society's Transactions, 1904 : read Dec. 20th, 1904.

† Wied. Ann. vol. lxvi. p. 49 (1898): Drude's Ann. vol. iii. p. 75 (1900).

†. Drude, Ann. vol. ix. p. 271 (1902).

\$ Proc. Roy. Soc. vol. lxiv. p. 377 (1899).

II Annales de Chimie et de Physique, 1897, p. 496.

If Proc. Camb. Phil. Soc. 1899, p. 217.

** Comptes Rendus, 1901, pp. 371, 734, 1286.

t+ When the work described in this paper was completed and the paper partly written, one on the same subject by Mr. Eve appeared in the Philosophical Magazine, Dec. 1904; the points discussed in the two papers are not, however, by any means always the sane. In cases where the same points are discussed the agreement is, on the whole, good, although in some cases somewhat different deductions have been made. 
protected. The radium is placed at $R$ so as to send a pencil of rays through a hole in a thick lead screen, other lead screens being placed to protect the tube $\mathrm{T}$ from direct radiation.

Fig. 1.

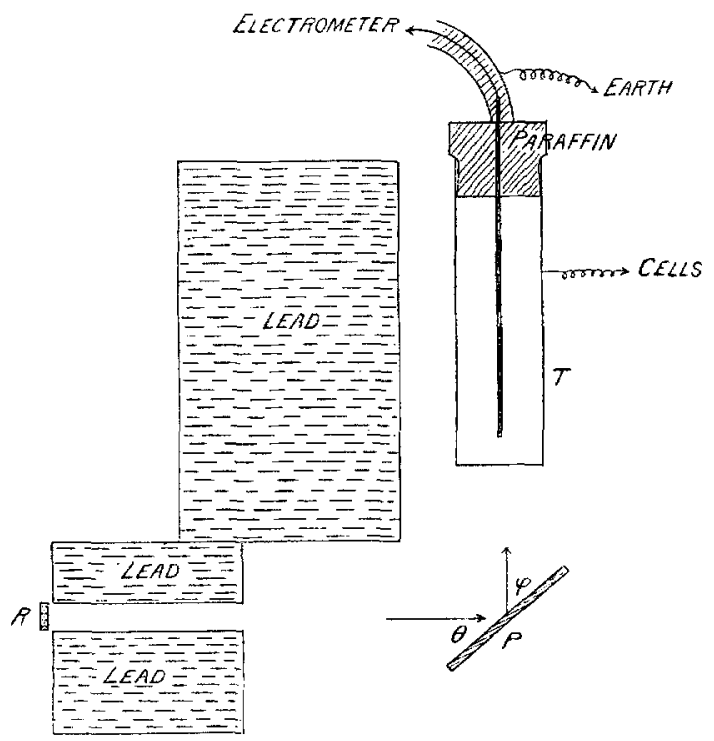

A plate of the substance under examination is placed at $\mathrm{P}$, and the secondary radiation from it enters the tabe $\mathrm{T}$ and produces ionization, which is measured in the usual way by the electrometer. The end of the tube $\mathrm{T}$ is covered with a single sheet of tinfoil. The distance from the radium to the plate $P$ was in most of the experiments about $26 \mathrm{cms}$. , and from $P$ to the nearest point of $T$ was usually about $9 \mathrm{cms}$. The distances are given in every case where they are of importance.

In every experiment the ionization observed in $\mathrm{T}$ is corrected for whatever small conductivity existed between the tube $\mathrm{T}$ and its inner terminal when the plate $\mathrm{P}$ is not in position; this small conductivity being due partly to the normal ionization inside $T$, and partly to insufficient screening of direct radiation from the radium, and also to secondary radiation from the air traversed by the primary rays.

Fifty milligrams of radium bromide were nsed, enclosed in a vessel which stopped the $\alpha$ radiation. 
IEffect of the Position of the Plate.

With the apparatus described it is easy to detect a secondary radiation which travels in all directions from the part of the plate struck by the primary rays. The first point investigated was whether the amount of secondary radiation depended on the angle of incidence of the primary rays, and on the angle that the testing-tube $T$ made with the plate; whether, in fact, there was anything of the nature of "reflexion" of the primary rays. This is a point which has been investigated by several observers using cathode rays as the primary rays; and while some observers have found a well-marked maximum of secondary radiation in the direction obeying the law of reflexion, others have not obtained such a result.

The point was first tested by keeping the direction of the primary rays and that of the tube $T$ constint and at right angles, as in the figure, while the lead plate $\mathrm{P}$ was tilted; and this experiment showed a well-marked maximum when $\theta$ was $45^{\circ}$.

The following observations were made, the secondary radiation being expressed in an arbitrary seale.

$$
\begin{aligned}
& \text { Secondary Radiation. } \\
& \begin{array}{l}
\theta=22 \frac{1}{2}^{\circ} \ldots \ldots \ldots \ldots \ldots \ldots \ldots \ldots \ldots \\
\theta=45^{\circ} \ldots \ldots \ldots \ldots \ldots \ldots \ldots \ldots \ldots \ldots \ldots \ldots \ldots \\
\theta=67 \frac{1}{2}^{\circ} \ldots \ldots \ldots \ldots \ldots \ldots \ldots \ldots
\end{array}
\end{aligned}
$$

The observed numbers are reduced so as to express the maximum by 100.

The plate $\mathrm{P}$ was then set so that $\phi$ was $45^{\circ}$, and this angle was kept constint, the direction of the primary rays only being changed. We then get as follows :-

$$
\begin{aligned}
& \theta=25^{\circ} \ldots \ldots \ldots \ldots \ldots \ldots \ldots . . \ldots 3 \\
& \theta=45^{\circ} \ldots \ldots \ldots \ldots \ldots \ldots \ldots \ldots
\end{aligned}
$$

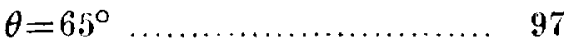

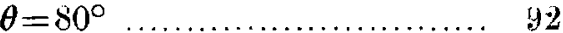

In the third case, the primary rays and the plate are both tilted, keeping the angle between them constant and equal to $45^{\circ}$, while the angle $\phi$ changes. We have then :-

Secondary Radiation.

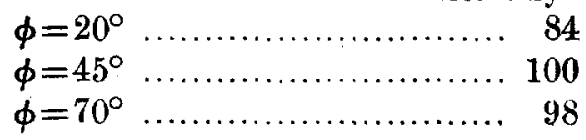

The first experiment shows a decided maximum when 
$\theta=45^{\circ}$. The other two experiments can be interpreted as showing a maximum when the law of reflexion is fulfilled, with the secondary radiation falling off unequally on the two sides of the maximum position.

The numbers indicate that, superposed on an effect of the nature of reflexion, we have other effects ; the law of reflexion being fulfilled, we get more secondary radiation the more we approach the normal, which is to be expected, as then the radiation coming from some depth in the plate has a smaller thickness to penetrate.

The general effect is therefore a radiation in all directions from the part of the plate struck by the primary rays, with a decided maximum in one direction, due probably to a sort of reflexion of the impinging particles. Many points described later show that the whole effect is not due to a simple scattering of the primary rays, but that it consists of a true secondary radiation from the plate.

It may also be stated here that experiments with a magnetic field, to be described later, show that the secondary rays we are now dealing with consist of negatively charged particles. The rays we are now dealing with travel through several centimetres of air, and also penetrate the tinfoil covering the end of the ionization-tube ' $\mathrm{T}$ before they produce the effect by which they are detected; there may be also a more easily absorbed radiation from the plate, which has not been studied in the present paper.

\section{Secondary Radiation not merely a Surface Effect.}

The secondary rays are not produced merely at the surface of the plate struck by the primary rays; it is easy to show that they come from all parts of a layer of considerable depth, the depth depending on the substance of which the plate is composed.

This point was tested by using for the plate in fig. 1 a single sheet of tinfoil (about $013 \mathrm{~mm}$. thick) in the first place, then two sheets, and so on. The secondary radiation was as follows:-

$\begin{array}{cc}\text { Sheets of Foil. } & \text { Secondary Radiation. } \\ 1 & 13 \\ 2 & 24 \\ 4 & 44 \cdot 5 \\ 8 & 60 \cdot 5 \\ 16 & 73 \cdot 5 \\ 32 & 77\end{array}$


Fig. 2 is plotted from these numbers.

Fig. 2.

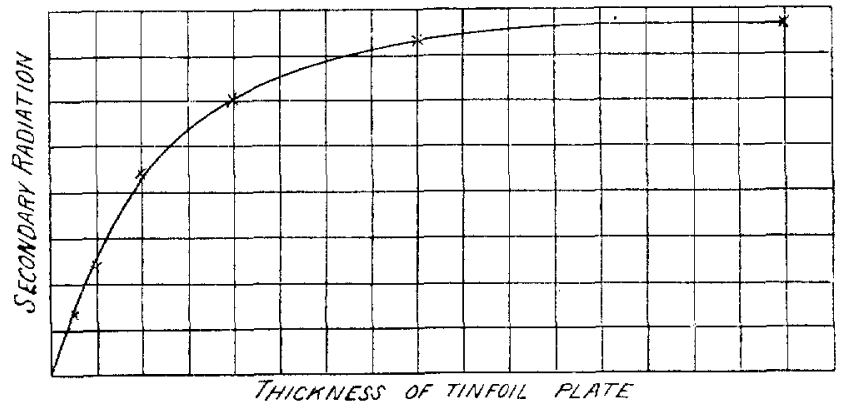

We should of course expect the secondary rays to come from all depths reached by the primary, unless the penetrating power of the secondary be less than that of the primary, in which case the effective thickness of plate will be determined by the penetrating power of the secondary.

The penetrating power of the secondary rays will be discussed later, but it may be pointed out here that the above numbers indicate that the secondary rays must at least approximate in penetrating power to the primary. The second sheet of tinfoil contributes almost as much secondary radiation as the first, and the rays from the second sheet must pass through the first before reaching the testing-tube $T$. The secondary rays must therefore suffer no very great loss of intensity in passing through the sheet of foil.

Analysing the Primary Radiation ( $\beta$ and y rays) with respect to its power of producing Secondary Rays.

The primary rays used in the above experiments were a pencil of $\beta$ and $\gamma$ rays : it is necessary to investigate what part of the secondary rays is due to the more penetrating part of the primary and what is due to the less penetrating primary rays.

This was done by making two separate experiments. In the first the apparatus was used as in fig. 1, and successive layers of tinfoil were placed in front of the lead screen so that the primary rays passed through them, and the intensity of the secondary rays was measured at each step.

In the second experiment, the radium with the thick lead screen was placed as in fig. 4 , so that a pencil of primary rays fell directly on the testing-tube $\mathrm{T}$ travelling parallel to its axis, and as before sheets of tinfoil were placed in front 
of the radium and the ionization measured at each stage. We thus get from one experiment the intensity of the primary rays after they have passed through various thicknesses of foil; and from the other experiment we get a measure of the secondary rays produced by the primary after passing through the same thickness of foil as before.

The results are as follows, the primary and secondary radiation being expressed so as to have a maximum of 100 in each case.

Sheets of foil.

0

1

2

4

8

16

32
Primary Radiation.

100

61

$47 \cdot 5$

40

33

$27 \cdot 5$

23
Secondary

Radiation.

100

56

41

31

19

10

Fig. 3.

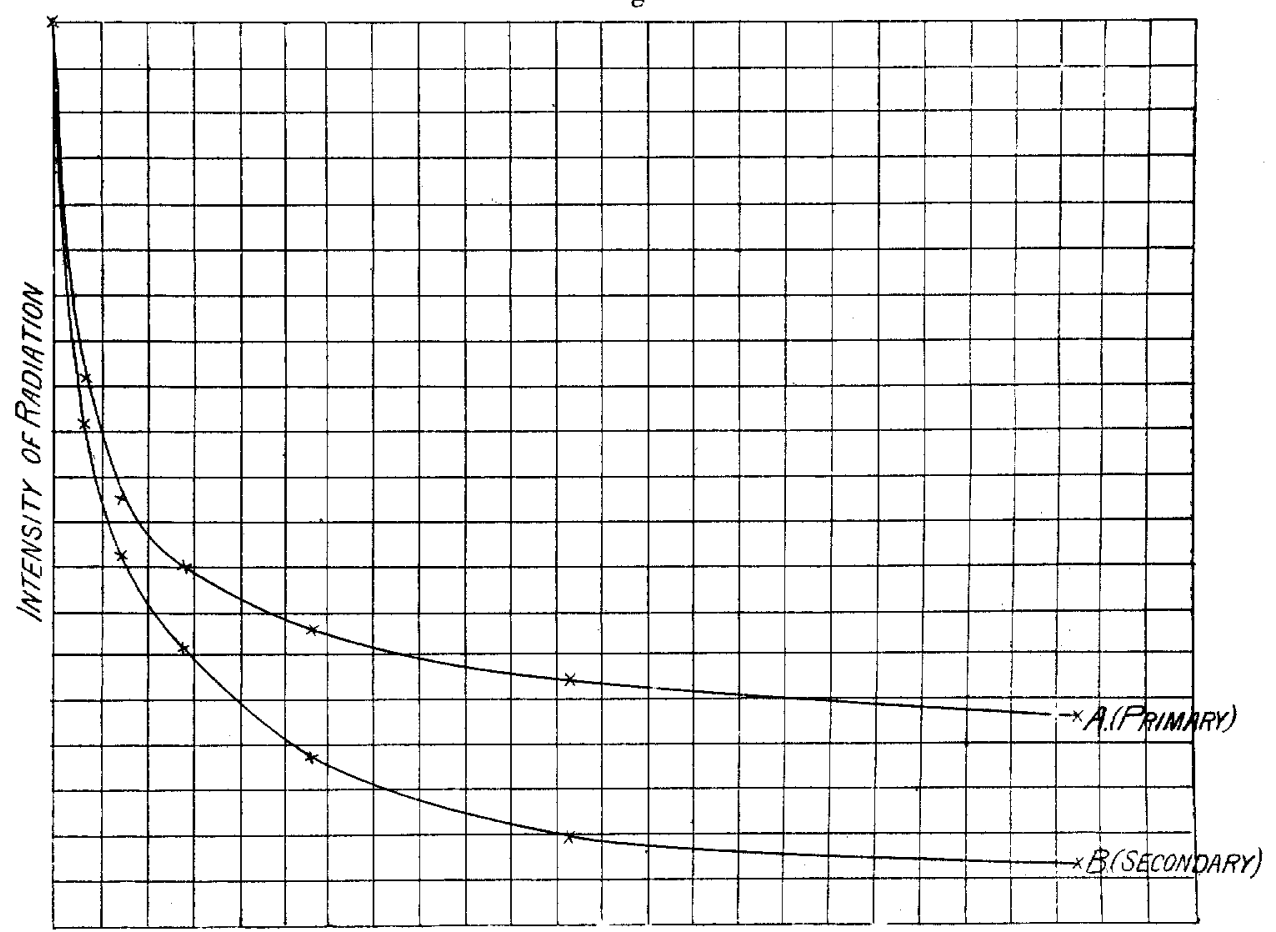

The curves in fig. 3 are plotted from the above numbers. The curves show at a glance the relative efficiency of the 
various parts of the primary pencil in producing secondary radiation. The less penetrating $\beta$ rays are more efficient than the more penetrating, and the efficiency falls off more and more as we get into that part of the curve $A$ corresponding to the $\gamma$ rays. But even when the curve $A$ has become practically horizontal-that is, when there is nothing left in the primary pencil but $\gamma$ rays-there is still some secondary radiation.

The magnetic experiments described later show that this part of the secondary radiation-the part produced by $\gamma$ rays -also consists of negatively charged particles.

\section{Different Substances as sources of Secondary Rays.}

The experiments described in this paper have all been carried out (unless when otberwise stated) with a lead plate as the source of secondary rays.

A large number of substances have been tested as sources of secondary rays. The substances were placed in the form of plates at the position $\mathrm{P}$, fig. 1 . The experiment described above in which tinfoil was used, shows that it is important when comparing substances to use in each case a thickness sufficient to give the maximum effect; this was done when comparing different substances.

The following list shows the results obtained, the radiation from lead being taken as 100 .

\begin{tabular}{|c|c|c|c|c|}
\hline Substance. & $\begin{array}{l}\text { Secondary } \\
\text { Radiation. }\end{array}$ & Density. & $\begin{array}{l}\text { Atomic } \\
\text { weight. }\end{array}$ & $\begin{array}{c}\text { Secondary } \\
\text { Radiation } \\
\text { Atomic weight }\end{array}$ \\
\hline 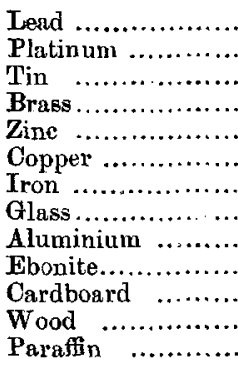 & $\begin{array}{r}100 \\
92 \\
80 \\
75 \\
65 \\
64 \\
62 \\
31 \\
33 \\
27 \\
28 \\
19 \\
18\end{array}$ & $\begin{array}{r}11 \cdot 4 \\
21 \cdot 5 \\
7 \cdot 3 \\
8 \cdot 4 \\
7 \cdot 2 \\
8 \cdot 9 \\
7 \cdot 8 \\
2 \cdot 5 \\
2 \cdot 7 \\
1 \cdot 15 \\
\cdot 80 \\
.52 \\
.90\end{array}$ & $\begin{array}{c}206 \cdot 4 \\
194 \cdot 3 \\
118 \cdot 8 \\
\\
65 \\
63 \cdot 2 \\
56 \\
\\
27\end{array}$ & $\begin{array}{r}\cdot 48 \\
\cdot 47 \\
\cdot 67 \\
1 \cdot 00 \\
1 \cdot 01 \\
1 \cdot 10 \\
1 \cdot 22\end{array}$ \\
\hline
\end{tabular}

The numbers show that, taken generally, the substances of greater density produce the greater secondary radiation; but there are many exceptions to the rule, and the secondary radiation is by no means proportional to the density. Platinum, 
for example, produces less radiation than lead, and tin more than copper.

It gives a more interesting result to compare the secondary radiation with the atomic weight of the substance: the atomic weight and the ratio of the secondary radiation to the atomic weight is given in the table. It will be seen that throughout the table the greater the atomic weight the greater is the secondary radiation. The ratio is not constant, but it will be noticed that the substances fall into groups with respect to this ratio. There is a well-marked group for which the ratio is unity, and another for which the ratio is one-half. Of course, the absolute value of the ratio is merely a question of the scale in which the secondary radiation is expressed.

It is intended to largely extend the list of substances examined, to see if the dependence of the radiation on the atomic weight holds throughout, and to follow out the apparent. grouping. The radioactive substances, radium, thorium, and uranium, are all substances of high atomic weight, and the results now before us indicate that the greater the atomic weight, the greater is the secondary radiation under the action of $\beta$ rays.

The secondary radiation seems therefore to be closely allied to the spontaneous radiation from radioactive substances. The impact of the $\beta$ rays produces a disturbance sufficient to cause the disintegration of the atom in substances which in the normal state are in stable equilibrium.

The dependence of the secondary radiation on the atomic weight and the close relation between it and radioactivity, suggests that the activity of radium might be increased by the action of its own radiations; and Voller (Physikalische Zeitschrift, Dec. 1, 1904) has obtained indications of such an action. We must remember, however, that the secondary radiation we are considering is one of $\beta$ particles, so that in extending our table to radium we should consider a transformation product of radium which emits $\beta$ particles.

It is interesting to compare the table given above with the results of observers who have studied the apparent small radioactivity of ordinary substances, when there is no artificially applied exciting cause. MeLennan found that lead was about twice as active as zinc, while tin gave an intermediate value; this is the same order as in our table of substances. A comparison with numbers given by Strutt for the activity of a number of substances does not, however, show much agreement.

In the above comparison of different substances, the secondary lays passed through a single sheet of tinfoil, $\cdot 013 \mathrm{~mm}$. 
thick; another set of observations was taken with the end of the tube $\mathrm{T}$ covered with a sheet of aluminium $3 \mathrm{~mm}$. thick in addition to the tinfoil. No difference in the order of the substances took place, but if we again express the secondary radiation from lead as 100 , the corresponding number for each substance is less than before when the rays passed through tinfoil only; the relative diminution is different for different substances, and is particularly great for ebonite, cardboard, and paraffin. In other words, of all the substances examined the secondary rays from lead are the most penetrating, those from paraffin, cardboard, and ebonite the least penetrating. The difference in penetrating power of the rays from the different substances is not very marked.

A rather rough experiment was tried to see if the temperature of the substance had any effect on its power of giving off this secondary radiation. A plate of copper was used, and its temperature increased by a strong bunsen-flame playing on the back of the plate; no change in the amount of secondary radiation was noticed. It should also be stated that no particular care was taken with the preparation of the surface of the different plates used; the surface was always well-cleaned, but the degree of polish was not attended to and did not seem to be of any importance.

As all numbers in this paper are given in arbitrary units, it may be useful to state a result specifically to give an idea of the absolute magnitude of the radiation we are dealing with ; 50 milligrams of radium bromide are placed $26 \mathrm{cms}$. from a lead plate as in fig. 1, the rays from the radium passing throngh a hole $1.2 \mathrm{cms}$. in diameter in a lead screen; the lead plate is $9 \mathrm{~cm}$. from the ionization-tube $\mathrm{T}$; the electrometer gives a deflexion of 2000 scale-divisions per volt, and the total capacity of the system charged is $\cdot 001$ microfarad; the secondary radiation then produces a deflexion of 115 scale-divisions per minute.

\section{Relative Importance of Secondary $\beta$ rays. Penetrating Power of Secondary $\beta$ rays.}

In making some experiments on the penetrating power of the secondary $\beta$ rays, and comparing it with that of the primary rays, some results were obtained which show that very different values may be deduced for the absorption of $\beta$ rays according to the apparatus used, and which also show very forcibly the importance of secondary effects when dealing with this type of radiation. Suppose we place the radium so that a pencil of $\beta$ (and $\gamma$ ) rays falls directly on 
the ionization-tube $\mathrm{T}$, as in fig. 4 . If now we proceed to measure the absorption produced by say a screen of cardboard, the result we get depends very much on where we place the cardboard in the path of the rays.

Fig. 4.
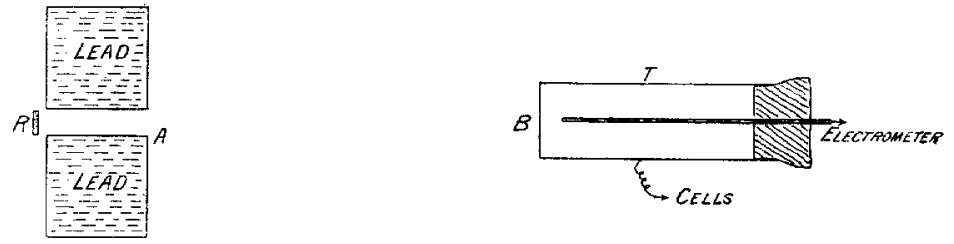

In an experiment the distance $\mathrm{AB}$, fig. 4 , was $26 \mathrm{cms}$, the internal diameter of the tube $\mathrm{T}$ being $3 \cdot 2 \mathrm{cms}$. The result was as follows:-

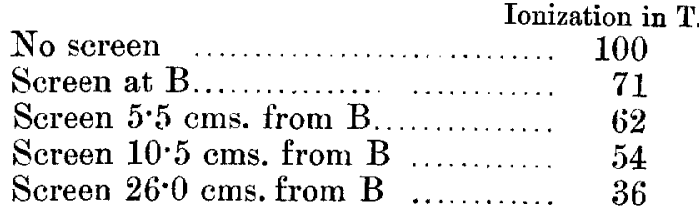

The screen produces a different effect in different positions, because it is a source of a radiation which travels in all directions from the part struck by the primary rays; and therefore the nearer the screen is to the testing-vessel $T$ the greater is the observed ionization. If the secondary rays were of much less penetrating power than the primary, the observed ionization would of course be increased by moving the screen towards the vessel $\mathrm{T}$; but we have seen there is no very great difference in penetrating power between the primary and secondary rays, so that the chief cause of the effect noted must be the fact that the screen becomes a source of radiation spreading out in all directions. Of course, we have also tertiary radiation from the air and from the end of the testing-tube.

The numbers given above show that after passing through the sheet of cardboard, the secondary rays are of as great importance as the primary rays, for the ionization with the screen close to the testing-vessel is twice what it is with the screen $26 \mathrm{cms}$. away.

Another example of the importance of the secondary rays is given by results obtained in comparing the penetrating power of the secondary rays with that of the primary pencil consisting chiefly of $\beta$ rays.

The radium was first placed as in fig. 4, and the primary 
rays examined by placing different thicknesses of tinfoil first at $B$ and then at $A$. The apparatus was then arranged as in fig. 1 , and the absorption of the secondary rays measured by placing sheets of tinfoil at the end of the tube $T$.

The results are as follows:-

\begin{tabular}{|c|c|c|c|}
\hline \multirow{2}{*}{$\begin{array}{l}\text { Sheets of foil. } \\
\text { Each } 013 \mathrm{~mm} \text {. thick. }\end{array}$} & \multicolumn{2}{|c|}{ Primary rays. } & \multirow{2}{*}{$\begin{array}{c}\text { Secondary rays. } \\
\text { 'Tinfoil at } \\
\text { ionization- } \\
\text { tube } \mathbf{T} \text {. }\end{array}$} \\
\hline & $\begin{array}{l}\text { Tinfoil } 26 \mathrm{cms} \\
\text { from ionization- } \\
\text { tube } \mathrm{T} \text {. }\end{array}$ & $\begin{array}{c}\text { Tinfoil at } \\
\text { ionization- } \\
\text { tube } \mathrm{T} \text {. }\end{array}$ & \\
\hline 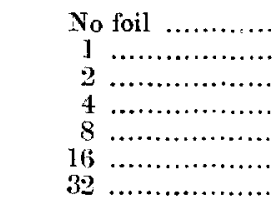 & $\begin{array}{r}100 \\
60 \\
47 \\
39 \\
32 \\
27 \\
22\end{array}$ & $\begin{array}{r}100 \\
96 \\
90 \\
77 \\
66 \\
49 \\
35\end{array}$ & $\begin{array}{r}100 \\
80 \\
64 \\
44 \\
26 \\
9\end{array}$ \\
\hline
\end{tabular}

Fig. 5.

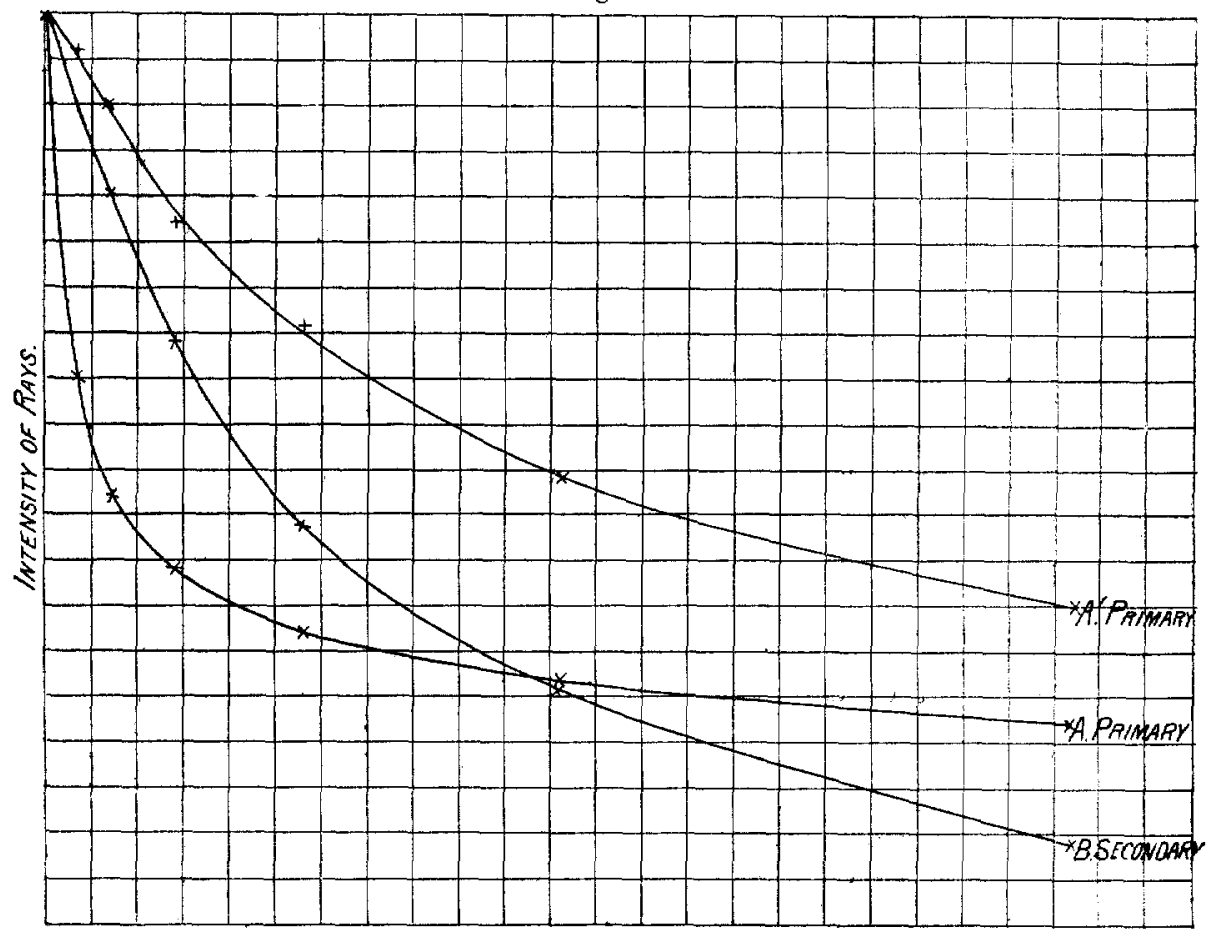

Curve A, fig. 5, refers to the primary rays when the absorbing tinfoil is placed $26 \mathrm{cms}$. from the ionization-tribe ; 
Curve $A^{\prime}$ to the primary rays with the foil placed close to the ionization-tube; Curve $B$ to the secondary rays. Curves $A$ and $A^{\prime}$ show the importance of the part played by the secondary rays, and how they may influence the value calculated for the coefficient of absorption. Comparing Curves $A^{\prime}$ and $B$, in both of which the foil is placed close to the ionization-tube, the secondary rays appear to be more easily absorbed than the primary; but the conditions of experiments are not quite the same in the two cases, as the secondary rays are not confined to a narrow pencil as in the case of the primary, and therefore the relative position of the absorbing tinfoil is not so important. The difference in penetrating power is probably, therefore, not so great as a comparison of the Curves $\mathrm{A}^{\prime}$ and $\mathrm{B}$ would suggest.

It will be observed that the primary Curves $A$ and $A^{\prime}$ become practically horizontal, which is of course due to the presence in the primary pencil of the very penetrating $\gamma$ rays. The secondary curve $\mathbf{B}$, on the other hand, continues to descend with increasing thicknesses of foil ; this is because the secondary pencil consists entirely of $\beta$ rays. The magnetic experiments showed that it was possible to deflect all the secondary rays. The experiments, therefore, did not detect any $\gamma$ rays produced by the stoppage of the primary $\beta$ particles, or by the starting of the secondary $\beta$ particles ; such rays may have existed in small amount and escaped detection, as we must remember that in every case a correction has to be made for the small normal ionization in the tube T.

The importance of the secondary rays in the above experiments suggest a more detailed consideration of the manner in which matter is penetrated by a stream of charged particles like $\beta$ rays; whether, in fact, there is great penetration by the primary particles, or whether it is not a successive stopping of one set and starting of another set of particles. The secondary rays from the above experiments are not very different in penetrating power from the rays that produce them, and the magnetic experiments described below indicate that the secondary rays contain some even more penetrating than the primary. The point is under further consideration.

\section{Magnetic Deflexion of the Secondary Rays.}

The nature of the secondary rays has been examined by passing them through a strong magnetic field. The apparatus (fig. 1, p. 231) was modified by interposing between the plate $\mathrm{P}$ and the ionization-vessel $\mathrm{T}$ the poles of an electromagnet so

Phil. Mrag. S. 6. Vol. 9. No. 50. Feb. $1905 . \quad$ K 
that the rays had to pass for a distance of $7 \cdot 7 \mathrm{cms}$. through a horizontal magnetic field; the total distance from plate $\mathrm{P}$ to vessel $T$ was $15 \mathrm{cms}$. If the secondary rays consist of charged particles, they should be deflected up or down and thrown off the end of the tube $T$. It was found that as the magnetic field was gradually increased the ionization in $\mathrm{T}$ gradually diminished, reaching a minimum which was not reduced by a further increase of the fièld. When this stage was reached, if the poles were allowed to come close together, thus interposing in the path of the rays a thickness of $7.7 \mathrm{cms}$. of iron, no further reduction of the ionization took place, showing that this residual ionization was not due to radiation from $P$, but simply to the normal ionization in $\mathrm{T}$, perhaps increased by some direct radiation from the radium. This residual ionization was therefore subtracted from that observed at each stage of increase of the magnetic field, and the remainder represented the effect due to the radiation from the lead plate $P$.

The result is shown in column 2 of the following table :-

\begin{tabular}{|c|c|c|}
\hline $\begin{array}{l}\text { Magnetic Field. } \\
\text { No Field... }\end{array}$ & $\begin{array}{l}\text { Secondary } \beta \text { Rays. } \\
\ldots \ldots \ldots 0\end{array}$ & $\begin{array}{c}\text { Primary } \beta \text { Rays. } \\
100\end{array}$ \\
\hline $160 \ldots$ & $\ldots \ldots \ldots$ & 81 \\
\hline 310 & 52 & 39 \\
\hline 460 & 23 & 12 \\
\hline 600 & 16 & 4 \\
\hline 960 & $\ldots \ldots$ & 0 \\
\hline 1250 & $\ldots \ldots$ & 0 \\
\hline 2300 & $\ldots \ldots$ & 0 \\
\hline
\end{tabular}

It was easy to show, by placing a thick lead screen in the path of the rays so as to cut off the upper or lower half of the pencil falling on the tube $T$, and then applying a magnetic tield first in one direction and then in the opposite, that the direction of deflexion of the rays showed them to be negatively charged. The fact that the residual ionization with strong magnetic fields was not diminished by interposing several centims. of iron, showed that there was no nondeflectable radiation of the nature of $\gamma$ rays, or at least that it was too small to be detected by the apparatus used.

The primary $\beta$ rays were examined in the same way, so as to get a comparison of the velocities of the primary and secondary, the mass and the charge being no doubt the same in the two cases. The radium with the accompanying lead screen was placed so that the primary rays fell directly on the tube $T$, passing between the poles of the magnet on the way. In this case a correction had to be applied for the 
$\gamma$ rays which could not be deflected, and for the normal ionization in $\mathrm{T}$.

The result is given in column 3 of the above table; the maximum when no magnetic field is applied is represented by 100 for both primary and secondary rays. The numbers show that while the first small field removes more secondary than primary, it requires a stronger field to remove coinpletely the secondary. The secondary pencil seems to include some which are travelling with a greater velocity than any of the primary.

XXIII. On the Theory of Electric Inertia.

By S. H. BurBUrY, F.R.S.*

1. SIR O. LODGE, in his address on Electrons read before $\$$ the Institution of Electrical Engineers, 1903, calculates the inertia which a small sphere, charged with electricity $e$, and moving with velocity $u$, has by virtue of its charge as a function of the charge. Inertia may be defined as resistance to acceleration. If the sphere have mass $m$ in the ordinary sense of that term, its kinetic energy if without charge is $\mathrm{E}=\frac{1}{2} m u^{2}$, and its inertia if it be moving freely is $2 \mathrm{E} / u^{2}=m$. But when charged it has electric energy in addition to $E$, namely,

$$
\mathrm{E}^{\prime}=\frac{\mu}{8 \pi} \int \mathrm{H}^{2} d \tau,
$$

where $\mathrm{H}$ is the magnetic force in the volume element $d \tau$ due to the motion of the sphere, $\mu$ is $a$ known constant, which I shall treat as unity, and the integration is throughout all space outside of the moving sphere. Further, he takes $\mathrm{H}=u e \frac{\sin \theta}{r^{2}}$, in which $r$ is the distance from $\mathrm{S}$, the present position of the sphere, to the point $P$ where the element $d \tau$ is, and $\theta$ is the angle between SP and tho axis, that is the direction of motion of the sphere. Substituting this value for $\mathrm{H}$, he obtains

$$
\mathrm{E}^{\prime}=\frac{e^{2} u^{2}}{4} \int_{c}^{\infty} d r \int_{0}^{\pi} d \theta \frac{\sin ^{3} \theta}{r^{2}}
$$

and the additional inertia due to the sphere being charged is in this theory $\frac{2 \mathrm{E}^{\prime}}{u^{2}}$, which is, as thus expressed, a function of $e$ and the radius $c$ of the sphere.

2. The value of $H$ here given is not generally exact, * Communicated by the Author. 